\title{
Investigation of the Performance of Filtration-charged Particles in a Reversed Electric Field
}

\author{
Yufeng Chang ${ }^{1}$, Pei Jia ${ }^{2 *}$, Xiaodong Xiang ${ }^{3}$, Ling Shi ${ }^{1}$, Xuepeng Jiang ${ }^{3}$ \\ ${ }^{1}$ Hubei Key Laboratory of Industrial Fume and Dust Pollution Control, Jianghan University, Wuhan 430056, China \\ ${ }^{2}$ School of Xingfa Mining Engineering, Wuhan Institute of Technology, Wuhan 430073, China \\ ${ }^{3}$ School of Resource and Environmental Engineering, Wuhan University of Science and Technology, Wuhan 430081, China
}

\begin{abstract}
Electrical forces can be applied to enhance fabric filters' ability to remove fine particles. To this end, we developed an experimental apparatus consisting of a conventional wire-tube particle precharger and fibrous filters positioned in a generated reversed external electric field. The charging and collection processes were separately accomplished in two stages, and we evaluated the device's ability to filter filtration-charged particles with a diameter of $\leq 2.5 \mu \mathrm{m}$. This device exhibited a higher electric field strength, higher collection efficiency, lower pressure drop, and lower electric potential than conventional devices due to the positioning of the wire and grounded electrodes close to the bag and the repulsion of the charged particles by the reversed electric field. When the face velocity was $2.5 \mathrm{~m} \mathrm{~min}^{-1}$, the collection efficiency for the charged particles with the reversed electric field was $8.4 \%$ and $64.4 \%$ higher than the efficiencies for the charged and uncharged particles, respectively, without the field. The charged particles also displayed a pressure drop when the field was applied that was 10\% lower and 5\% higher than those of the uncharged and charged particles, respectively, when the field was absent. A negative direct current supply was necessary to direct the deposition of the charged particles, and neither a spark discharge nor a back corona was observed while using the reversed-electric-field apparatus, which, according to our results, enables the removal of filtration-charged particles at face velocities beyond the usual range.
\end{abstract}

Keywords: Filtration; Particle precharger; Reversed external electric field; Collection efficiency.

\section{INTRODUCTION}

Particulate matter (PM), occurring mainly from power plants and industrial processes, has become the dominant pollutant in most major cities of China (Ni et al., 2018; Yao et al., 2009). In particular, PM with a diameter of $\leq 2.5 \mu \mathrm{m}$ potential threats both for human health and for the atmospheric environment (Dockery, 2009; Xie et al., 2016). New and rigorous ambient air quality standards have been devised and have necessitating novel and effective methods (Noh et al., 2001; Peukert and Wadenpohl, 2001; Deng et al., 2010; Sheng and Shen, 2017; Sobczyk et al., 2017; Chang et al., 2018; Sirignano and Danna, 2018) to remove fine particles from air to meet these standards.

Bag filters (BFs) and electrostatic precipitators (ESPs) are widely used due to their high collection efficiency; their total mass collection efficiency is typically $>99 \%$ (Goryachev, 1981; Mizuno, 2000; Deng et al., 2010; Zhang et al., 2018;

\footnotetext{
${ }^{*}$ Corresponding author.

Tel.: 86-27-87193718

E-mail address: ddjp1980@wit.edu.cn
}

Boudhan et al., 2019). However, a single BF or ESP has little ability to capture fine particulates; for fine particle removal, combinations of conventional BFs and ESPs have received considerable attention (Kanaoka et al., 2001; Christofides et al., 2007; Bologa et al., 2009; Park et al., 2012; Boudhan et al., 2018; Wang et al., 2018). Over 1996-2006, the compact hybrid particulate collector and the advanced hybrid particulate collector have been developed as typical representatives of this hybrid approach (Chang, 1996; Hrdlicka and Swanson, 2006; Miller and Schelkoph, 2001). Electrically energized fibrous filters constitute the theoretical basis of the hybrid BF-ESP technology. Electrostatic force can be applied on fibrous filters by precharging the particles, by collecting the particles in an electric field, or by using a combination of both (Jaworek et al., 2007).

For high collection efficiency, high electric potential can be applied on electrodes in an electric field maintained at a high level; such devices tend to exhibit sparking discharges and back coronas. Several remedies have been proposed and tested to minimize sparking discharge and back corona (Ivancsy et al., 2009; Krupa et al., 2019).

This paper describes the development of a laboratoryscale, electrically energized, fibrous-filter device, which includes particle precharger and fibrous filters in a reversed 
electric field. The collection principle and collection efficiency of this system was investigated.

\section{THEORY}

\section{Conventional External Electric Field}

When a fibrous filter was placed between the inner elements and an external electric field, notable filtration results were obtained for the wire electrodes located on the dusty side of the filter (Long et al., 2009), as shown in Fig. 1. The ionic current was generated similarly to the flowing gas, which was defined as conventional external electric field.

For optimization of surface area and bag cleaning, the dusty gas usually flows from the outside of the bag to the inside of the bag in industrial applications, as depicted in Case 2. The wire electrodes are typically located far away from the bag to prevent sparking and back corona. The longer the distance between the wire electrode and the bag, the lower is the electric field on the bag surface; thus, electrodes are placed far away to prevent sparking and back corona tend to produce devices with poor electrostatic effects (Mermelstein et al., 2002; Podgórski and Balazy, 2008; Sanchez, 2013; Tu et al., 2016).

\section{Reversed External Electric Field}

In a typical two-stage electrostatic precipitator with electrically energized fibrous filters, the charging and collection processes can be separated; these two types of processes are accomplished in two stages (Jaworek et al., 2017; Jaworek et al., 2018). Regardless of whether the wire electrode is located in dusty or clean gas, the main function of the collection stage can be maintained at high potential; accelerated charged particles are deposited on the surface of the bag. A model of filtration-charged particles in a reversed electric field was developed, as illustrated in Fig. 2. The ionic current was generated in opposition to the flowing gas, which was defined as reversed external electric field.

Fig. 2 illustrates the stainless-steel wire cage, which was comprised of framework and ring clamps to fix the bag, was

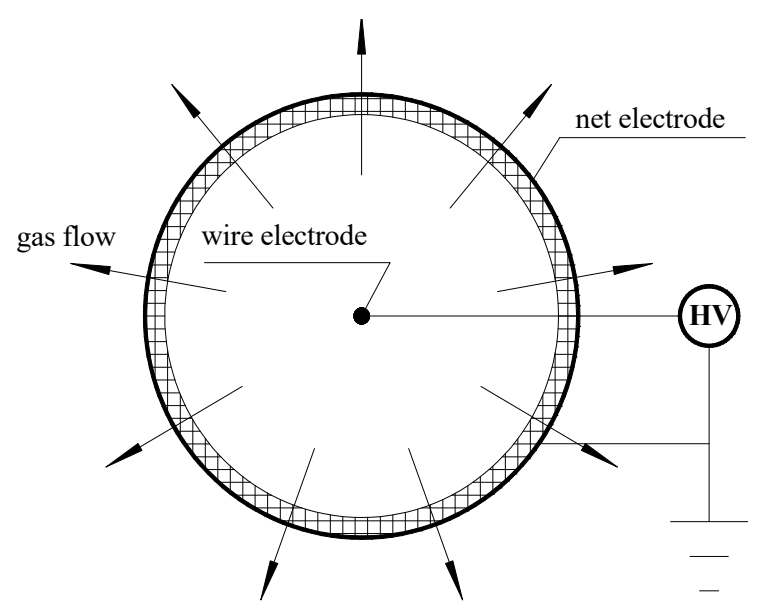

(a) maintained at high potential; the outside surface of the bag was grounded. The dusty gas flowed from the outside to the inside of the bag to maximize surface area. The wire electrodes were located in clean gas to optimize cleaning.

\section{Theoretical Velocity of Charged Particles}

The Coulomb force of a charged particle in an electric field is:

$F=q E$

where $F$ is the Coulomb force $(\mathrm{N}), q$ the particle charge $(\mathrm{C})$, and $E$ the electric field $\left(\mathrm{V} \mathrm{m}^{-1}\right)$.

The viscous force on a moving particle in the gas flow was given by:

$$
f=\frac{3 \pi \mu d_{p}\left(u_{0}-\omega\right)}{C_{m}}
$$

where $\mu$ is the viscosity $(\mathrm{Pa} \cdot \mathrm{s}), d_{p}$ the diameter of particle $(m)$,

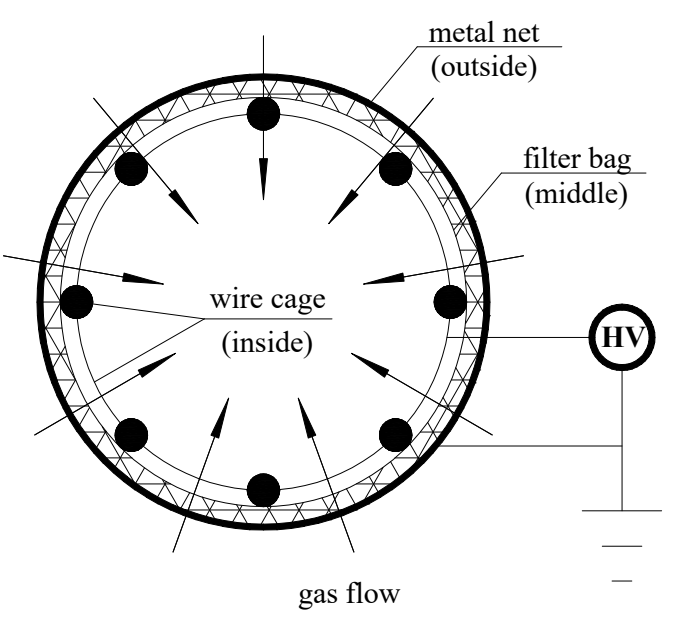

Fig. 2. Schematic of reversed electric field (Case 3).

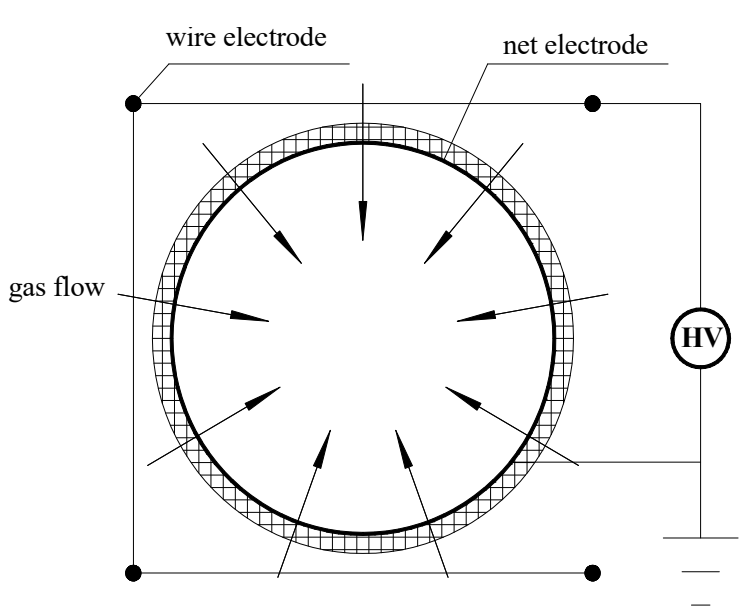

(b)

Fig. 1. Schematic of wire electrodes located at the dusty side: gas flow from (a) inside to outside (Case 1) and (b) outside to inside (Case 2). 
$u_{0}$ was the velocity of gas flow $\left(\mathrm{m} \mathrm{s}^{-1}\right), \omega$ the velocity of particle $\left(\mathrm{m} \mathrm{s}^{-1}\right)$, and $C_{m}$ the Cunningham correction factor.

In Fig. 1, the charged particles are moved toward the filter bag by Coulomb force, so the velocity of a charged particle in the conventional external electric field can be written as:

$\omega_{e}=u_{0}+\frac{q E C_{m}}{3 \pi \mu d_{p}}$

However, in Fig. 2, the charged particles are moved against the filter bag by Coulomb force, and the velocity of a charged particle in the reversed external electric field can be written as:

$\omega_{r}=u_{0}-\frac{q E C_{m}}{3 \pi \mu d_{p}}$

It is empirically true that:

$\omega_{r}<\omega_{e}$

In a $\mathrm{BF}$, the lower the velocity of a charged particle, the higher is the collection efficiency.

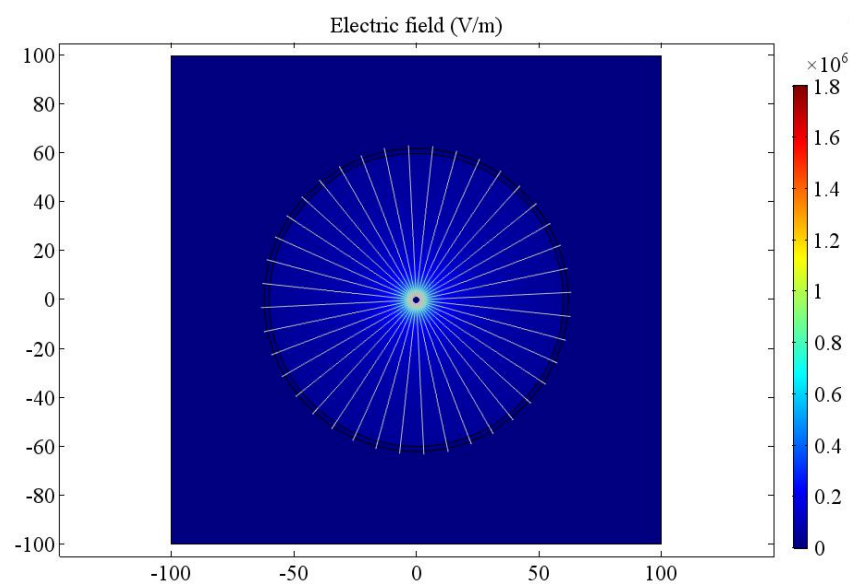

(a) Case 1

\section{Distribution of the Electric Field}

It is difficult to get the distributions of the electric fields directly. Therefore, commercial software COMSOL Multiphysics 5.2 was used for the solution of electric field based on Poisson equation.

The distributions of the electric fields at different types of electrodes are depicted in Fig. 3, and the distribution of the electric field strength on middle section of the filter bag is shown in Fig. 4, where the diameter of the filter bag is $120 \mathrm{~mm}$, the electric potential of Case 1 and Case 2 is $-10 \mathrm{kV}$, and the electric potential of Case 3 is $-1.0 \mathrm{kV}$.

Fig. 4 illustrates experiments in which a high electric field strength was achieved on the filter bag in a reversed electric field, although the applied potential was $1 / 10$ that of the conventional electric field. In addition to energy saving, this apparatus avoided sparking discharge because of its low electric potential.

\section{EXPERIMENTAL}

The measurements were carried out with the experimental setup shown schematically in Fig. 5. The experimental setup was composed of a conventional wire-tube particle precharger and fibrous filters in a reversed electric field. The stainlesssteel tube had a diameter of $100 \mathrm{~mm}$, and the stainless-steel

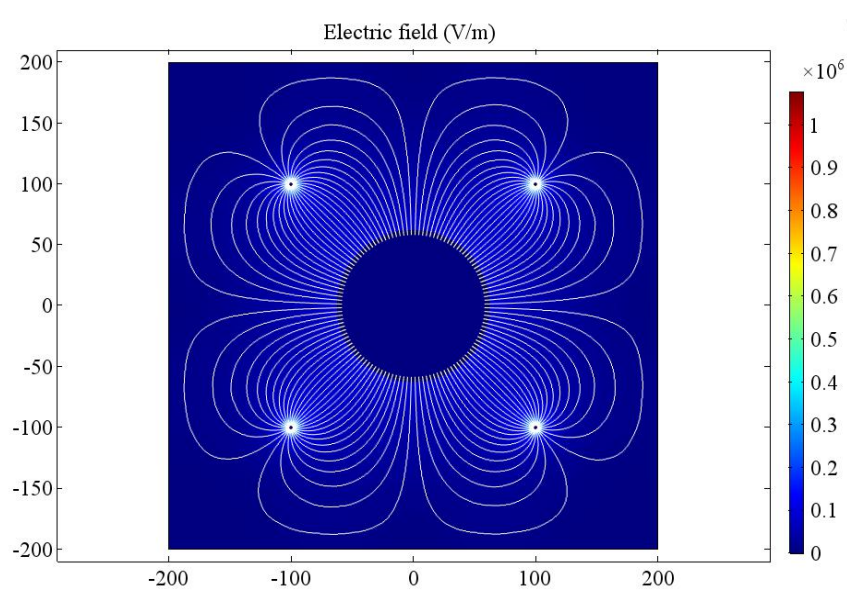

(b) Case 2

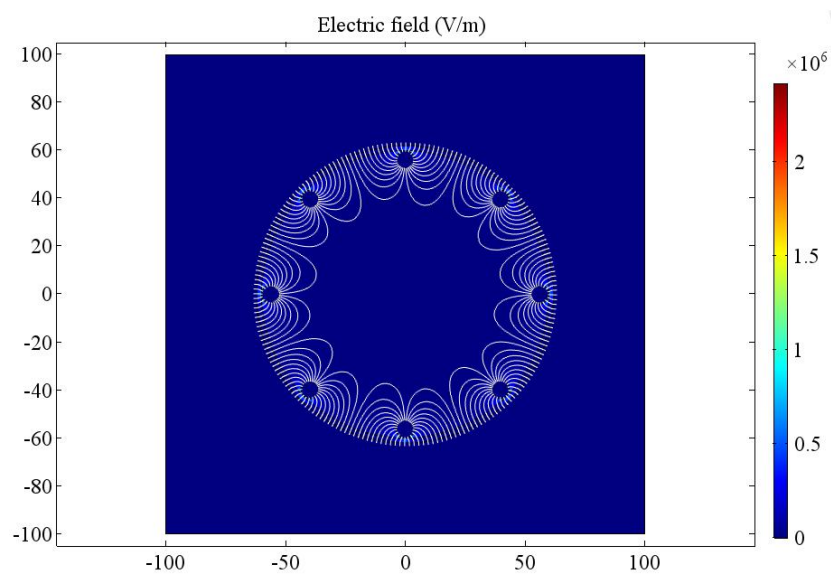

(c) Case 3

Fig. 3. Distributions of the electric fields at different types of electrodes: Cases (a) 1, (b) 2, and (c) 3. 


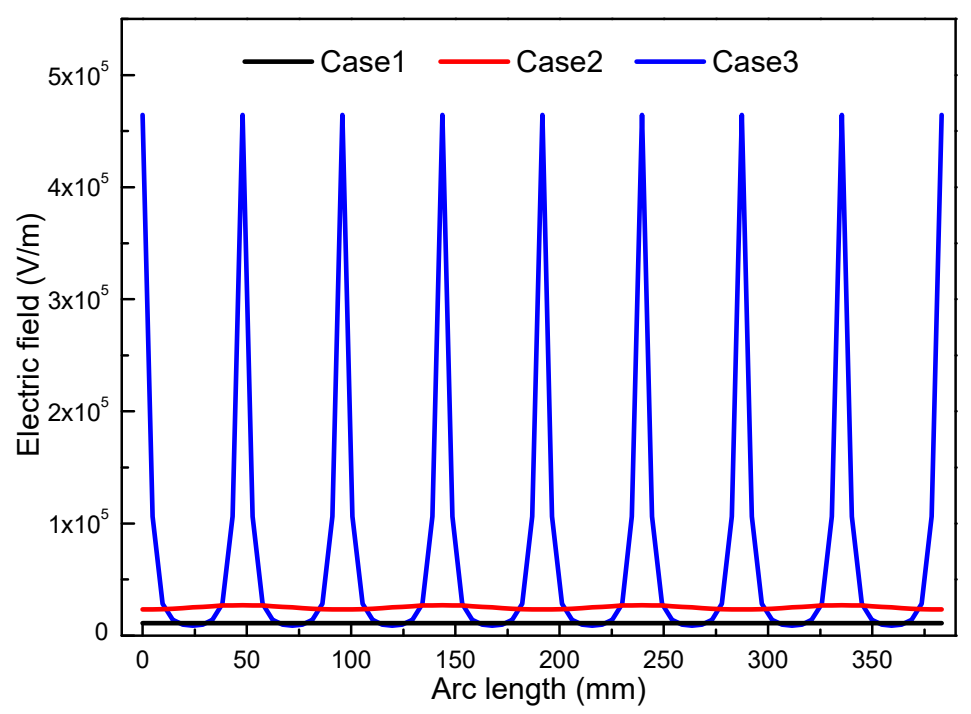

Fig. 4. Comparison of electric field strength at different electrodes (middle section of the filter bag).

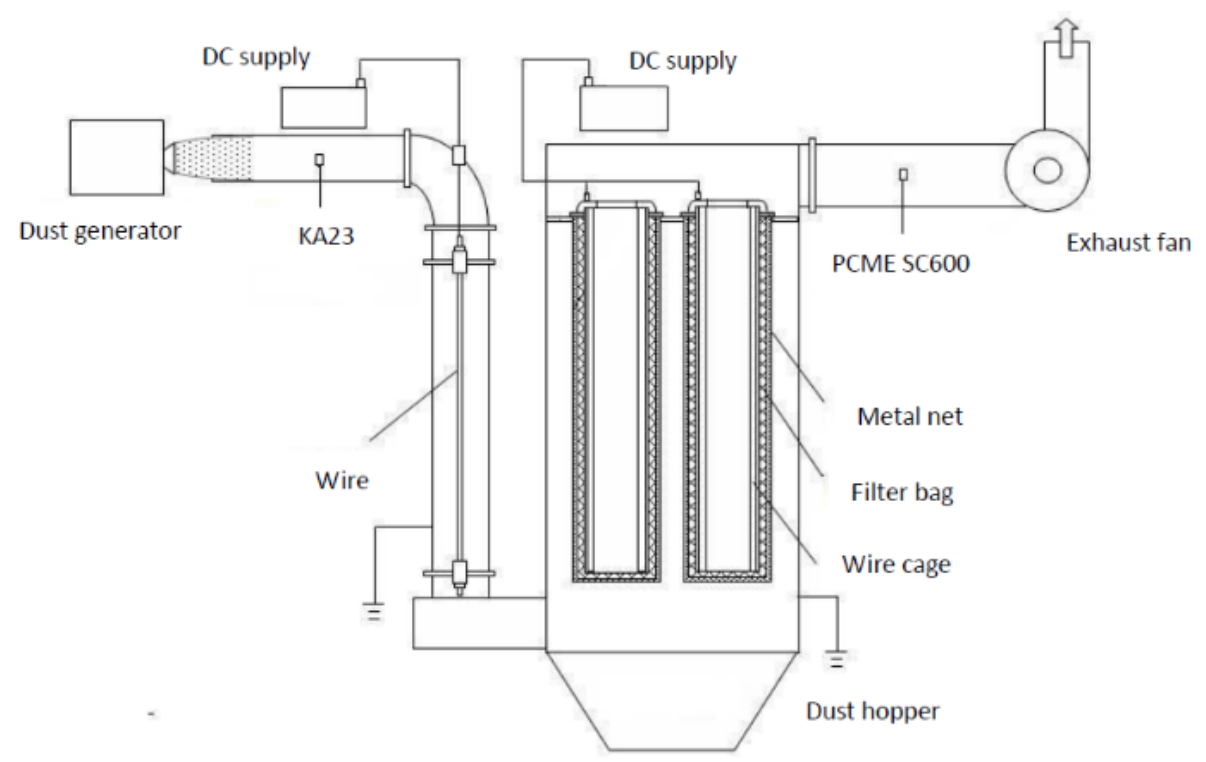

Fig. 5. Schematic of experimental setup.

corona wire was $2 \mathrm{~mm}$ in diameter and $1,000 \mathrm{~mm}$ in length. The collection stage consisted of a polymethyl methacrylate (PMMA) channel of square cross section, of $450 \times 450 \mathrm{~mm}^{2}$, and dust hopper was located at the bottom. An exhaust fan was mounted at the outlet of the channel to control the air flow rate. The aerosol, which produced by dust generator, was injected to the channel at its inlet. Particle concentration was measured at the outlet of the channel by using a particle measurement device. The air flow velocity in the channel was measured by a hot-wire anemometer.

The precharger consisted of a wire-tube electrode and a DC supply. The space between the wire and the stainlesssteel tube was $50 \mathrm{~mm}$. The DC supply connected the wire electrode to a high-voltage power supply, and the tube was grounded.

The collection stage was consisted of a filter bag, wire cage (inside), metal net (outside), and DC supply. Filtration was in four circular acetate fiber bags, which were $120 \mathrm{~mm}$ in diameter, $1000 \mathrm{~mm}$ in length, and $1.55 \mathrm{~m}^{2}$ in total filter surface area. The stainless-steel wire cage consisted of eight cylindrical skeletons and several 8-mm-diameter rings. Stainless-steel netting, which was used to replace the bags with conductive yarns in the experiment, was fixed on the outside surface of every fiber bag. The space between the stainless-steel wire cage and the stainless-steel net was approximately $2 \mathrm{~mm}$, which was the thickness of the circular acetate fiber bags. By DC supply, the wire cage was connected to high-voltage power supply model $-60 \mathrm{kV} / 5 \mathrm{~mA}$ and $+60 \mathrm{kV} / 5 \mathrm{~mA}$, and the metal net was grounded. The collection efficiency of filtration-charged particles in the reversed electric field was measured at four filter face velocities, namely $1.0,1.5,2.0$, and $2.5 \mathrm{~m} \mathrm{~min}^{-1}$.

Total collection efficiency of fibrous filters in a reversed electric field was determined from the measurements of 
particle concentration in the $i^{\text {th }}$ class when the voltages were switched ON. The fibrous filters were loaded at a value of $c_{1}$ when the voltages were switched OFF. The fibrous filters were unloaded at a value of $c_{0}$. The particles were sampled only at the outlet of the channel.

$\eta_{t}(i)=1-\frac{c_{1}(i)}{c_{0}(i)}$

If any relevant collection efficiency is close to unity, the performance can be accurately characterized with the outlet dust loading by penetration $\lambda$, which can be defined as:

$\lambda(i)=1-\eta_{t}(i)=\frac{c_{1}(i)}{c_{0}(i)}$

Three filtration models were tested in the experimental setup:

1) Filtration-uncharged particles: The voltages of both the precharger and collection stage were OFF.
2) Filtration-charged particles: Precharger voltage was $\mathrm{ON}$ and collection-stage voltage was OFF.

3) Filtration-charged particles in reversed electric field: The voltages of both the precharger and collection stage were $\mathrm{ON}$.

\section{RESULTS AND DISCUSSION}

\section{Particle Size Distribution}

The particle size distribution of the dust in the laboratory was measured using a laser particle size analyzer. As shown in Fig. 6, the median particle size was approximately $1.49 \mu \mathrm{m}$, and the particles with diameters of $\leq 1.92 \mu \mathrm{m}$ contributed approximately $90 \%$.

\section{V-I Characteristics of Particle Precharger and Collection Stage}

The results of the $\mathrm{V}-\mathrm{I}$ characteristics of particle precharger and collection stage are given in Fig. 7.

The results indicated that the inception voltage value of the particle precharger was approximately $10 \mathrm{kV}$ and that of the collection stage was approximately $200 \mathrm{~V}$, regardless of

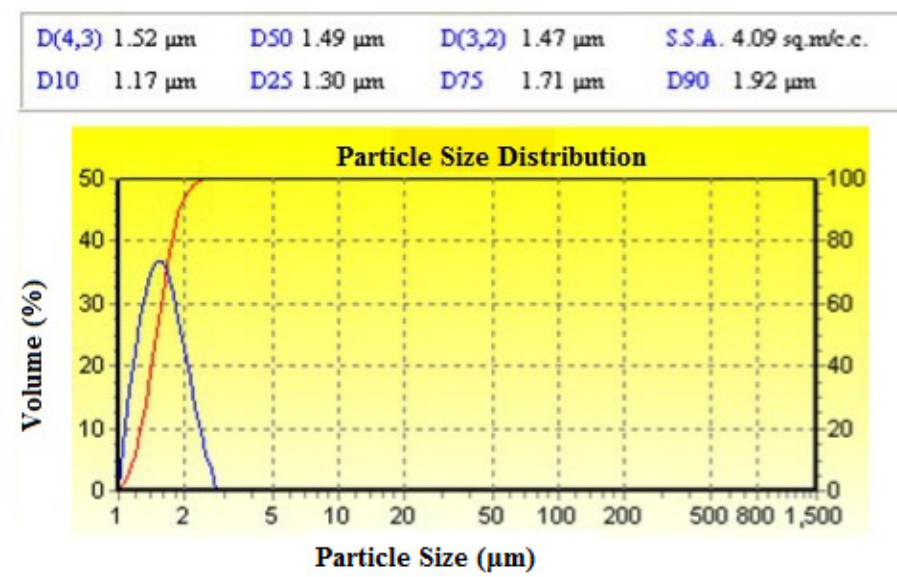

Fig. 6. Particle size distribution of the dust.

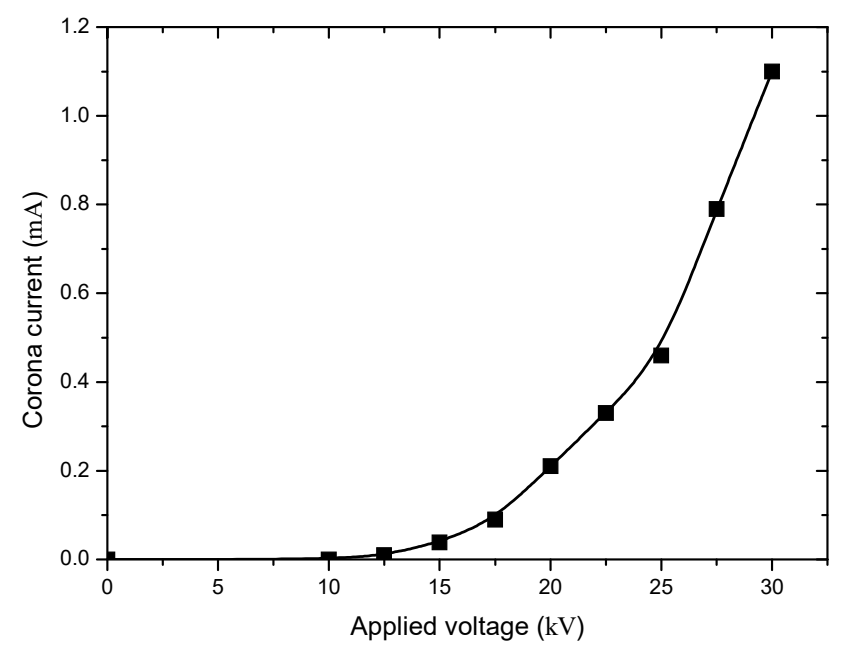

(a)

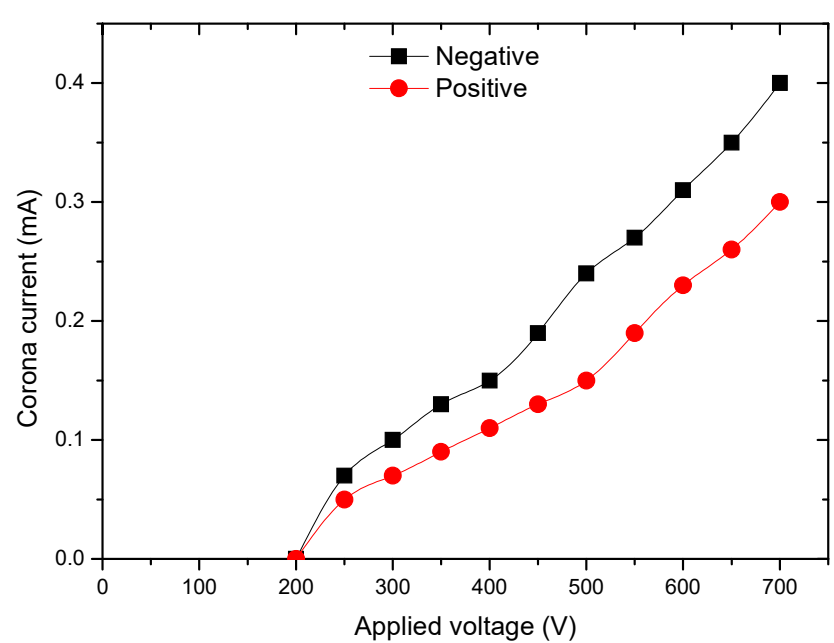

(b)

Fig. 7. V-I characteristics of the experimental setup: (a) particle precharger and (b) collection stage. 
whether negative or positive DC was applied. When negative DC was applied on the collection stage, the corona current was approximately $25-30 \%$ higher than that of the positive DC supply. The radius of cylindrical skeletons, which were used discharge electrodes in collection stage, was greater than that of the discharge electrodes in particle precharger. Therefore, the V-I characteristic curve of the collection stage was gentler than that of the particle precharger.

Based on our previous work, the DC supply voltage of the particle precharger was $18 \mathrm{kV}$ and the mean electric field strength was approximately $3.6 \mathrm{kV} \mathrm{cm}$. The DC supply voltage of the collection stage was set as 400-600 V, and the mean electric field strength was approximately 2.0 $3.0 \mathrm{kV} \mathrm{cm}^{-1}$.

\section{Effect of Different Electrostatics Added to Fibrous Filters on Collection Efficiency}

When the DC supply voltage of collection stage was -400 $\mathrm{V}$, the collection efficiency of filtration-charged particles in the reversed electric field was measured, relative to that of filtration-uncharged and -charged particles, as shown in Fig. 8.

Fig. 8 illustrates an experiment in which the collection efficiency of charged particles in the reversed electric field was higher than that of filtration-charged particles and that of filtration-uncharged particles. The lower the face velocity, the higher were the collection efficiency values of different kinds of fibrous filters. The decline rate of charged particles in a reversed electric field was slower than that of filtrationcharged particles and that of filtration-uncharged particles. When the face velocity increased from 1.0 to $2.5 \mathrm{~m} \mathrm{~min}^{-1}$, the collection efficiency of charged particles in a reversed electric field decreases by approximately $7.9 \%$; however, that of filtration-charged particles and that of filtrationuncharged particles decrease by approximately $11.7 \%$ and $39.9 \%$ respectively.

When face velocity was $2.5 \mathrm{~m} \mathrm{~min}^{-1}$, the collection efficiency of charged particles in a reversed electric field was $8.4 \%$ and $64.4 \%$ higher than that of filtration-charged and -uncharged particles, respectively. The advantage of the reversed electric field was significant for high face velocities. The higher the face velocity is, the smaller is the size and the smaller is the number of the BF. High face velocity can be useful for collecting charged particles in a reversed electric field in industrial applications.

\section{Effect of the Polarity of External Electric Field on Collection Efficiency}

Fig. 9 presents reversed-field charged-particle collection efficiency curves for DC supply voltage values of -400 and $+400 \mathrm{~V}$ in the collection stage.

Fig. 9 illustrates that when a negative DC supply was applied on collection stage, the collection efficiency was 5$15 \%$ higher than that of the positive DC supply. Therefore, in the setup of charged particles in a reversed electric field, one of the highest priorities was the negative DC supply, which was consistent with the conventional electrically energized fibrous filters.

The particles, negatively charged in the precharger stage, flowed through the collection stage. When negative DC supply was applied on the collection stage, a negative polarity electric field was generated. The negatively charged particles were rejected by the electrostatic force from the filter layer. The dust was deposited on the surface of the fabric rather than on the fibers. Thus, regeneration of BFs was simple and efficient.

When a positive DC supply was applied, a positive polarity electric field was generated. The negatively charged particles were accelerated by electrostatic force into the filter layer. A positive result was that large particles were captured in the collection stage due to electrostatic agglomeration. A negative result was that the dust cake in the filter was difficult to remove.

\section{Effect of Electric Field Strength on Collection Efficiency}

Fig. 10 illustrates an experiment in which the DC supply voltage of collection stage was from $-400 \mathrm{~V}$ to $-600 \mathrm{~V}$; the vertical axis corresponds to the collection efficiency of charged

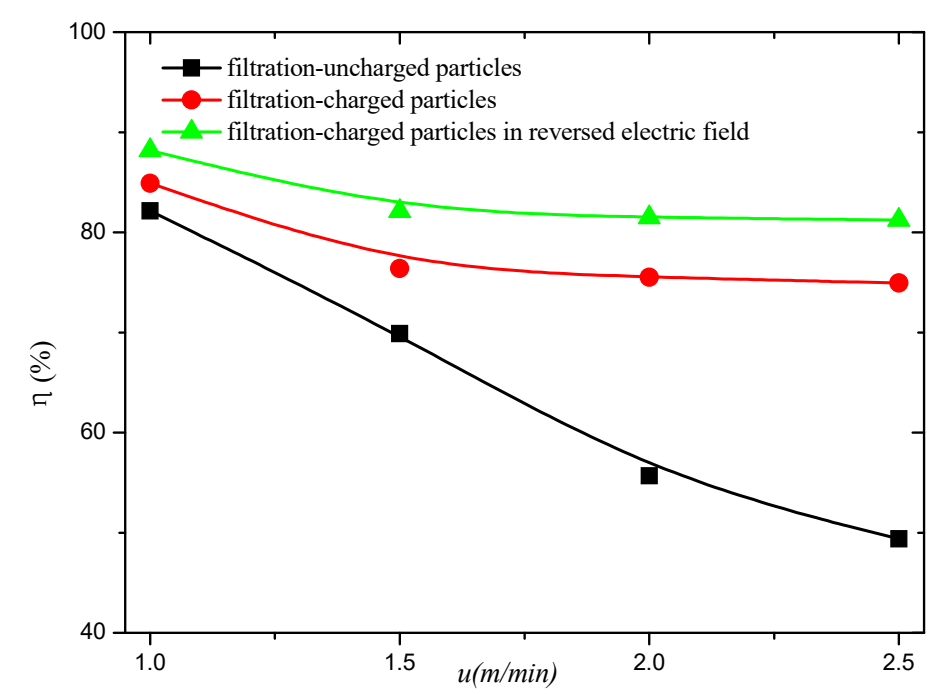

Fig. 8. Collection efficiency of different electrostatic materials added to fibrous filters. 


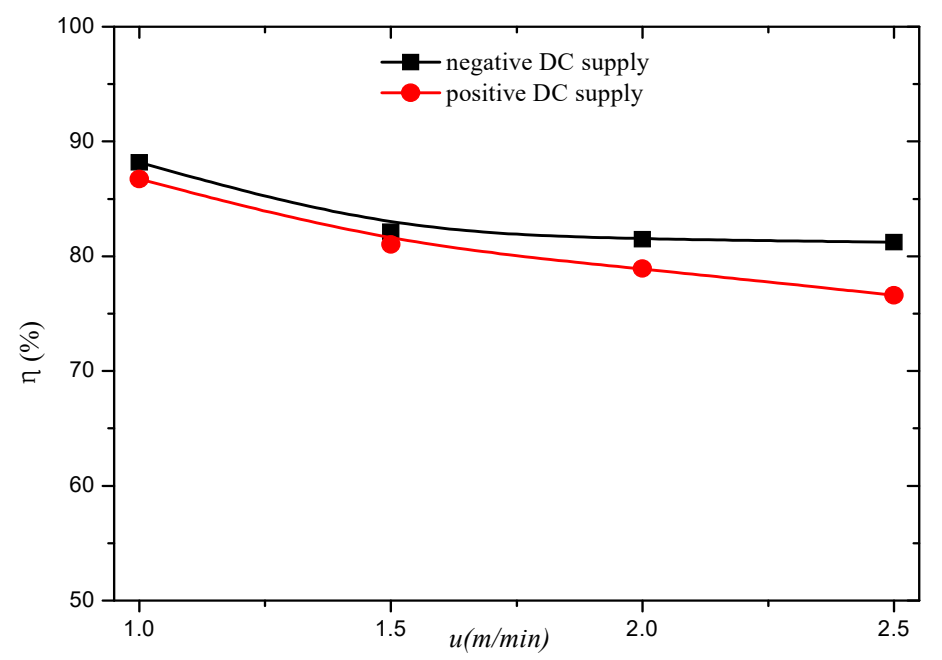

Fig. 9. Collection efficiency of different polarity DC supply added to collection stage.

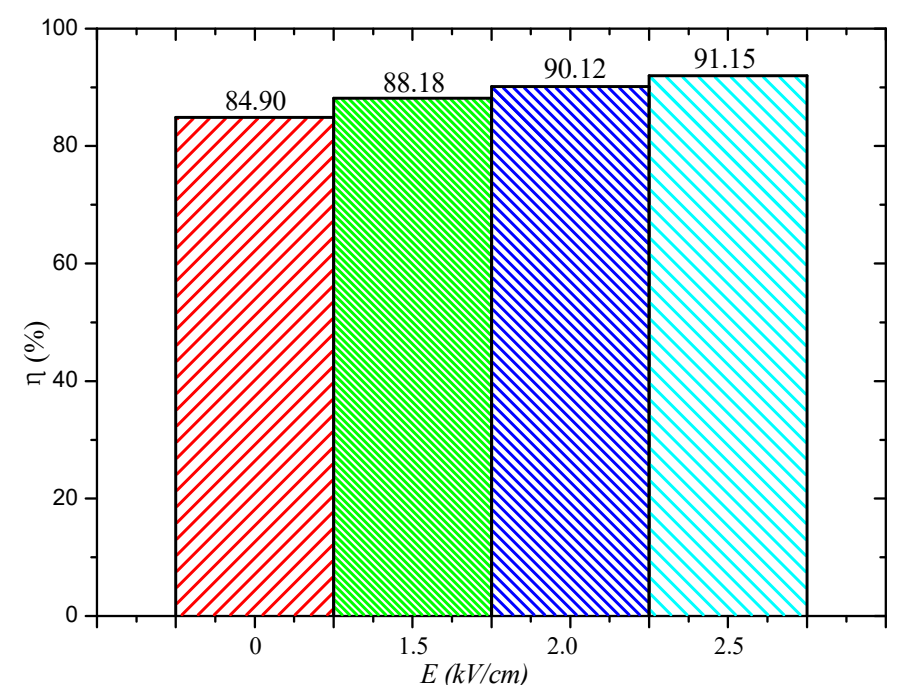

Fig. 10. Collection efficiency of different electric field strength of collection stage.

particles in a reversed electric field for a specific face velocity of $1.0 \mathrm{~m} \mathrm{~min}^{-1}$. The corresponding mean electric field strength of collection stage was from 2.0 to $3.0 \mathrm{kV} \mathrm{cm}^{-1}$.

In Fig. 10, the higher the collection-stage electric field strength is, the higher the collection efficiency is. When the DC supply voltage of collection stage was from $-400 \mathrm{~V}$ to $-600 \mathrm{~V}$, the collection efficiency of charged particles in a reversed electric field was $3.8-8.3 \%$ higher than that of filtration-charged particles. A positive result was that the penetration of charged particles in the reversed electric field was $21.7-46.7 \%$ lower than that of filtration-charged particles.

High collection efficiency and low corona energy consumption were achieved by these charged particles in this reversed electric field.

\section{Pressure Drop}

The measuring results of pressure drop are indicated in Fig. 11.

Fig. 11 illustrates an experiment in which the pressure drop of charged particles in a reversed electric field was $10 \%$ lower than that of filtration-uncharged particles and 5\% higher than that of filtration-charged particles. The pressure drop declined when electrostatic materials were added to fibrous filters because charged particles tend to form a notably porous dust cake on the surface of the filter bag (Amiri et al., 2016). The negative polarity electric field of the collection stage rejected the negatively charged particles; thus, the pressure drop of charged particles in that reversed electric field was higher than that of filtration-charged particles.

\section{CONCLUSIONS}

In this study, the collection efficiency of a laboratoryscale apparatus generating a reversed electric field to enhance the removal of filtration-charged particles was determined for different configurations. The following conclusions can be drawn:

1) Because the wire and grounded electrodes were positioned close to the bag of charged particles, high electric field 


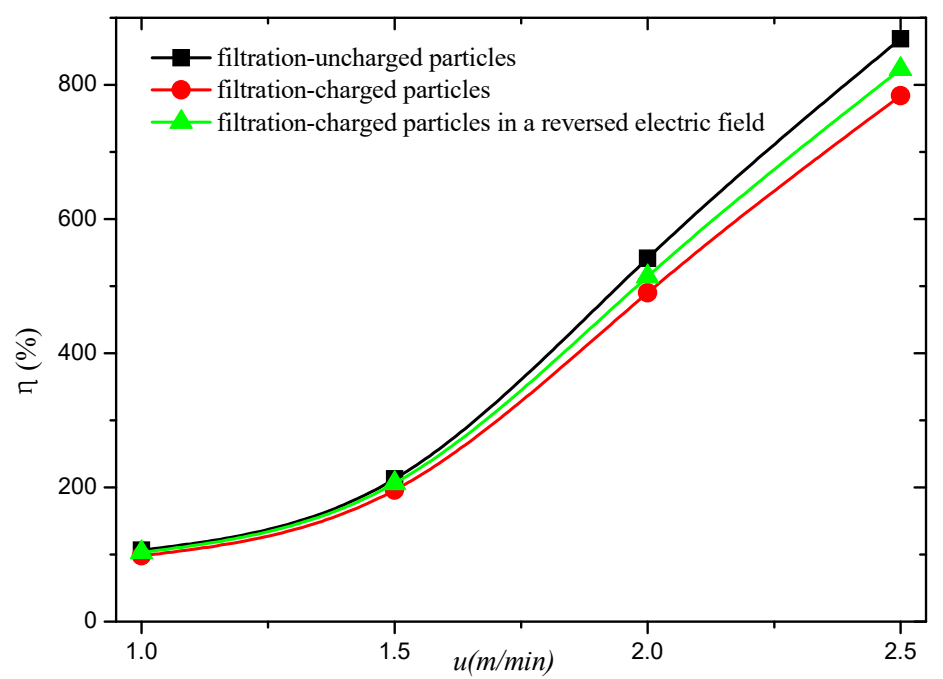

Fig. 11. Pressure drop of different electrostatic materials added to fibrous filters.

strength and collection efficiency were achieved without modifying the surface area or the bag cleaning mode. Furthermore, to eliminate spark discharges and back coronas, a low electric potential was used.

2) The collection efficiency slowly declined as the face velocity increased. The gas flow rate can be increased without changing the collection efficiency, which allows the size or the number of bags used for filtration to be reduced.

3) Because the negatively charged particles were rejected by the electrostatic force of the filter layer, a negative DC supply was crucial for directing the deposition of the particles. The collection efficiency increased with the electric field strength during the collection stage.

4) The action of the electric field caused the deposited dust to become flocculent. Thus, the pressure drop declined when electrostatic materials were added to the fibrous filters.

\section{ACKNOWLEDGEMENT}

Financial support from the 2019 Hubei Province Technical Innovation Special Major Project (2019ACA160) and Hubei Key Laboratory of Industrial Fume \& Dust Pollution Control open project (HBIK2019-03) were gratefully acknowledged. This manuscript was edited by Wallace Academic Editing.

\section{REFERENCES}

Amiri, M., Sadighzadeh, A. and Falamaki, C. (2016). Experimental parametric study of frequency and sound pressure level on the acoustic coagulation and precipitation of $\mathrm{PM}_{2.5}$ aerosols. Aerosol Air Qual. Res. 16: 3012-3025.

Bologa, A.D., Paur, H.R., Seifert, H., Wascher, T. and Woletz, K. (2009). Novel wet electrostatic precipitator for collection of fine aerosol. J. Electrostat. 67: 150-153.

Boudhan, R., Joubert, A., Gueraoui, K., Durecu, S., Venditti, D., Tran, D. and Coq, L.L. (2018). Pulse-jet bag filter performances for treatment of submicronic and nanosized particles from waste incineration. Waste Biomass Valorization 9: 1-7.

Boudhan, R., Joubert, A., Durecu, S., Gueraoui, K. and Coq, L.L. (2019). Influence of air humidity on particle filtration performance of a pulse-jet bag filter. J Aerosol Sci. 130: 1-9.

Chang, R. (1996). COHPAC compacts emission equipment into smaller, denser unit. Power Eng. 100: 22-25.

Chang, Y., Jia, P., Shi, L. and Xiang, X. (2018). Corona discharging and particle collection of bipolar transverse plate ESP. J. Electrostat. 96: 104-110.

Christofides, P.D., Li, M. and Madler, L. (2007). Control of particulate processes: Recent results and future challenges. Powder Technol. 175: 1-7.

Deng, H., He, Z., Ma, J., Xu, Y., Liu, J. and Guo, R. (2010). Initiation and propagation of discharge in liquid droplets: Effect of droplet sizes. IEEE Trans. Plasma Sci. 38: 3282-3288.

Deng, H., He, Z., Xu, Y., Ma, J., Liu, J. and Guo, R. (2010). An investigation on two-phase mixture discharges: The effects of macroparticle sizes. J. Phys. D 43: 255203.

Dockery, D.W. (2009). Health effects of particulate air pollution. Ann. Epidemiol. 19: 257-263.

Goryachev, I.K. (1981). Bag filters for cleaning up industrial gases. Chem. Pet. Eng. 17: 624-626.

Hrdlicka, T. Swanson, W. (2006). Demonstration of a fullscale retrofit of the advanced hybrid particulate collector technology. Final report. Otter Tail Power Company.

Ivancsy, T., Kiss, I. and Berta, I. (2009). Improved model for the analysis of back corona in pulse energised electrostatic precipitators. J. Electrostat. 67: 146-149.

Jaworek, A., Marchewicz, A., Sobczyk, A.T., Krupa, A. and Czech, T. (2017). Two-stage electrostatic precipitator with co- and counter-flow particle prechargers. J. Electrostat. 87: $180-194$.

Jaworek, A., Marchewicz, A., Sobczyk, A.T., Krupa, A. and Czech, T. (2018). Two-stage electrostatic precipitators for the reduction of $\mathrm{PM}_{2.5}$ particle emission. Prog. Energy 
Combust. Sci. 67: 206-233.

Jaworek, A., Krupa, A. and Czech, T. (2007). Modern electrostatic devices and methods for exhaust gas cleaning: A brief review. J. Electrostat. 65: 133-155.

Kanaoka, C., Hiragi, S. and Tanthapanichakoon, W. (2001). Stochastic simulation of the agglomerative deposition process of aerosol particles on an electret fiber. Powder Technol. 118: 97-106.

Krupa, A., Podlinski, J., Mizeraczyk, J. and Jaworek, A. (2019). Velocity field of EHD flow during back corona discharge in electrostatic precipitator. Powder Technol. 344: 475-486.

Long, Z., Yao, Q., Song, Q. and Li, S. (2009). Threedimensional simulation of electric field and space charge in the advanced hybrid particulate collector. J. Electrostat. 67: $835-843$.

Mermelstein, J., Kim, S. and Sioutas, C. (2002). Electrostatically enhanced stainless steel filters: Effect of filter structure and pore size on particle removal. Aerosol Sci. Technol. 36: 62-75.

Miller, S. and Schelkoph, G. (2001). Advanced hybrid particulate collector, a new concept for air toxics and fine particle control. National Energy Technology Lab. (NETL), USA, DOE/PC/95258, doi: 10.2172/766247.

Mizuno, A. (2000). Electrostatic precipitation. IEEE Trans. Dielectr. Electr. Insul. 7: 615-624.

Ni, Z., Luo, K., Gao, Y., Gao, X., Fan, J. and Cen, K. (2018). Potential air quality improvements from ultralow emissions at coal-fired power plants in China. Aerosol Air Qual. Res. 18: 1944-1951.

Noh, K., Lee, J., Kim, C.K., Yi, S., Hwang, J. and Yoon, Y. H. (2011). Filtration of submicron aerosol particles using a carbon fiber ionizer-assisted electret filter. Aerosol Air Qual. Res. 11: 811-821.

Park, B.H., Kim, S.B., Jo, Y. and Lee, M. (2012). Filtration characteristics of fine particulate matters in a PTFE/glass composite bag filter. Aerosol Air Qual. Res. 12: 1030 1036.

Peukert, W. and Wadenpohl, C. (2001). Industrial separation of fine particles with difficult dust properties. Powder Technol. 118: 136-148.

Podgórski, A. Balazy, A. (2008). Novel formulae for deposition efficiency of electrically neutral, submicron aerosol particles in bipolarly charged fibrous filters derived using Brownian dynamics approach. Aerosol Sci. Technol. 42: 123-133.

Sanchez, A.L. (2013). Experimental study of electrostatic aerosol filtration at moderate filter face velocity. Aerosol Sci. Technol. 47: 606-615.

Sheng, C. and Shen, X. (2007). Simulation of Acoustic agglomeration processes of poly-disperse solid particles. Aerosol Sci. Technol. 41: 1-13.

Sirignano, M., Danna, A. (2018). Filtration and coagulation efficiency of sub-10nm combustion-generated particles. Fuel 221: 298-302.

Sobczyk, A.T., Marchewicz, A., Krupa, A., Jaworek, A., Czech, T., Śliwiński, Ł., Kluk, D., Ottawa, A. and Charchalis, A. (2017). Enhancement of collection efficiency for fly ash particles $\left(\mathrm{PM}_{2.5}\right)$ by unipolar agglomerator in two-stage electrostatic precipitator. Sep. Purif. Technol. 187: 91-101.

Tu, G., Song, Q. and Yao, Q. (2016). Relationship between particle charge and electrostatic enhancement of filter performance. Powder Technol. 301: 665-673.

Wang, C., Tu, T., Syu, J., Kuo, C., Kuo, P., Jhong, Y. and Lin, W. (2018). Experimental investigation of the filtration characteristics of charged porous fibers. Aerosol Air Qual. Res. 18: 1470-1482.

Xie, Y., Dai, H., Dong, H., Hanaoka, T. and Masui, T. (2016). Economic impacts from $\mathrm{PM}_{2.5}$ pollution-related health effects in China: A provincial-level analysis. Environ. Sci. Technol. 50: 4836-4843.

Yao, Q., Li, S., Xu, H. W., Zhuo, J. and Song, Q. (2009). Studies on formation and control of combustion particulate matter in China: A review. Energy 34: 1296-1309.

Zhang, W., Deng, S., Wang, Y. and Lin, Z. (2018). Dust loading performance of the PTFE HEPA media and its comparison with the glass fibre HEPA media. Aerosol Air Qual. Res. 18: 1921-1931.

Received for review, July 5, 2019 Revised, September 27, 2019 Accepted, October 22, 2019 